\title{
HIV-depression: A Shadow Over 90-90-90 UNAIDS Program
}

\author{
Jorge L Zirulnik*, Maria Sol Retamar, Hector M Perez
}

HIV/Infectious Diseases Division, JA Fernandez Hospital, Buenos Aires, Argentina

Article Info

\section{Article Notes}

Received: July 04, 2018

Accepted: July 28, 2018

\section{${ }^{*}$ Correspondence:}

Dr. Jorge Zirulnik, HIV/Infectious Diseases Division, JA Fernandez Hospital, Buenos Aires, Argentina, Email: jorzirulnik@gmail.com

( 2018 Zirulnik JL. This article is distributed under the terms of the Creative Commons Attribution 4.0 International License.
There are 36.9 million people living with HIV (PLWHIV) according to a 2017 WHO estimation; $70 \%$ of the affected population is living in low and middle-income countries (LMIC). Depression, alcohol/ substance abuse and neurocognitive deficit are the three most common psychiatric disorders found in PLWHIV in said countries. Depression is the most prevalent, with a rate over $30 \%$. It is of high interest then, to study the way in which this high prevalence has an impact on initiatives for the prevention and treatment of HIV infection, such as the 90-90-90 program of UN-OMS. The way to do this, is to focus on the current state of the depression treatment cascade observed in PLWHIV, and its link with adherence to antiviral treatment ${ }^{1}$.

As expressed in the Corless IB et al. investigation (2017): Medication adherence is the "Plus" in the global challenge to have $90 \%$ of HIV-infected individuals tested, $90 \%$ of those who are HIV positive treated, and 90\% of those treated achieve an undetectable viral load [90-90-90 UN AIDS proposal for 2020]. This group found that, self-efficacy, depression, stressful life events and perceived stigma were significant predictors of medication adherence. According to our clinical observations, depression is one of the leading risk factors causing adherence failure to antiretroviral treatment; however, it is a risk factor that can be reversed if properly treated ${ }^{2}$. As seen in the literature, the cascade associated with the diagnosis and treatment of HIV-depression appears to be in a truly discouraging state:

A. In the estimation of Pence BW et al. based on several studies: (...) of all HIV patients with MDD [Major Depressive Disorder], 18\% (14$24 \%)$ are receiving any treatment, $7 \%$ (4-12\%) are receiving adequate treatment, and 5\% (3-9\%) have achieved remission. See (Figure 1) ${ }^{3}$.

B. HIV-depression management in relation to the strategic 9090-90 program, consists in its insertion within a continuum that goes from detection and correct treatment, executed with evidencebased interventions, to obtaining remission, and not merely the improvement of symptoms. A multicenter study carried out by the Cholera R group in the USA has revealed an unfavorable scenario to this continuum, both in cross-sectional analysis and prospective observations. In the cross-sectional design, the number of patients who received adequate treatment did not reach $20 \%$, while only $9 \%$ achieved remission. In the prospective study, merely $12 \%$ of evidence-based treatments were identified, with only $7 \%$ of remissions. A significant gap can be seen in these studies, both in the detection and the rational treatment of the HIV-psychiatric depressive condition that concerns us. See (Figure 2$)^{4}$. 


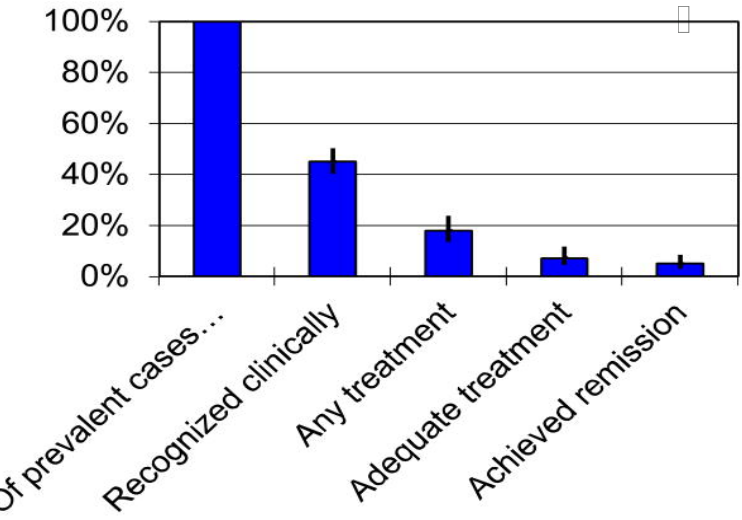

Figure 1: The depression treatment cascade for HIV patients The figure depicts, of all HIV patients with a major depressive episode in the past year, the estimated proportion whose depression is recognized clinically, who are receiving any treatment, who are receiving adequate treatment, and who have achieved remission.

B Pence, O’Donnell JK, Gaynes BN. AIDS 2012, 26(5): 636-637

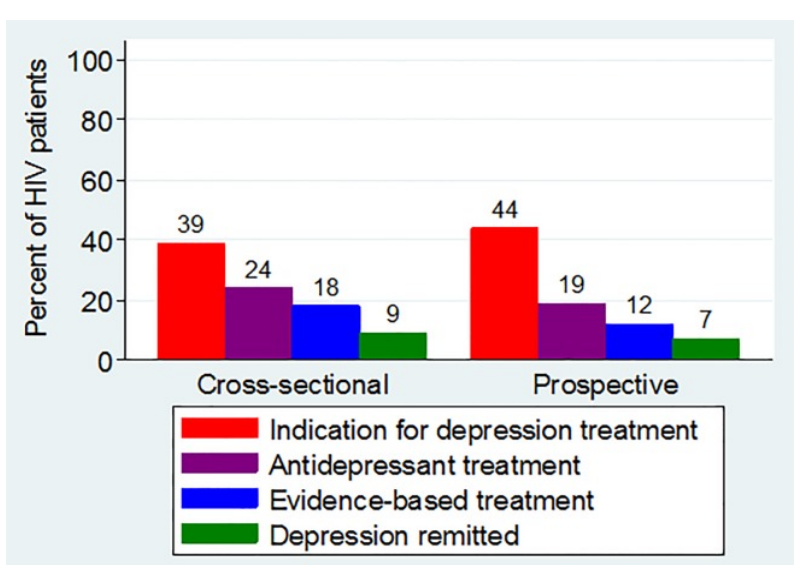

Figure 2: The depression treatment continuum for patients engaged in HIV primary care

R Cholera, Pence BW, Bengtson Am, et al. PLoS one 2017, 12(1): e016643

If we take into account that there is already strong evidence that treatment of HIV-depression favors adherence to antiretroviral therapy, we believe that a conceptual modification should be added to the continuum under consideration: in the criteria established for remission of HIV associated depression, full adherence to antiretroviral treatment should be included [95\%]. Thus, when dealing with HIV positive patients, the only acceptable remission of the depressive state is one that includes full adherence to ARVs; that is to say completion of antiretroviral treatment must be guaranteed in the process. We believe that for this purpose, it should be mandatory to add an intensive, brief course of cognitive-behavioral psychotherapy on ARV adherence, of no more than eight weeks' duration, to the already established antidepressant evidence-based management ${ }^{5-7}$. See (Figure 3).

\section{Fig 3}

\section{HIV TREATMENT}

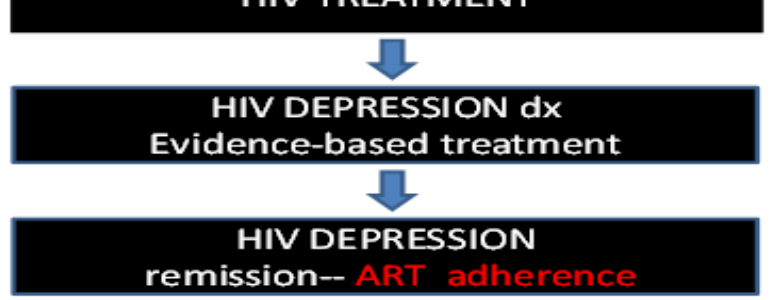

Figure 3: The new perspective of HIV-depression treatment final outcome

It is essential to analyze intervention models in mental health and HIV, in order to better understand how to bridge this gap. There are three models, which have not yet been compared to each other in clinical trials: the classic model based on consultation-liaison psychiatry; the model of immersion or in-setting, and the so-called "Medical Home" model. Speculatively, it can be said that these models differ in their accessibility, integration, attrition and eventual impact on the 90-90-90 system $^{6-10}$.

The consultation-liaison model is based on a referring system of PLWH to an external psychiatry service, separated from the HIV unit, and with psychiatrists who usually have little preparation for the assistance of these patients. They even tend to fall into situations that reinforce the associated HIV stigma, damaging as a result the continuum of the process ${ }^{7}$.

The in-setting model considers the mental health team as a member of the HIV unit, along with the infectious diseases physicians. This model is very demanding for psychiatrists who work in an integrated manner with the HIV clinicians and its effectiveness needs to be proved in clinical essays ${ }^{8-9}$.

In the Medical Home system, non-professional personnel are used - although supervised by a psychiatrist - to obtain a faster access to the treatment of HIV associated depression, thus not interfering with the continuum. This model has a paradox: it is hard to implement in low resource HIV clinics, such as Latin America's, due to cultural prejudices based on the belief that non-professional health providers do not have proved skills to face complex tasks involving PLWH. Table 1 shows a comparative scheme of these models ${ }^{9,10}$.

To conclude this brief editorial, our intention is to draw attention to the three critical points regarding the current state of HIV depression and its negative impact on the 9090-90 initiative:

a. Although depression is the most prevalent psychiatric condition in HIV patients, its detection, adequate treatment and resolution are far from being achieved. In many cases, 
Table 1. Table comparing HIV Mental Health Models of assistance in LMIC

\begin{tabular}{|c|c|c|c|}
\hline & $\begin{array}{c}\text { Consultation- } \\
\text { liaision }\end{array}$ & In-setting & $\begin{array}{c}\text { "Medical } \\
\text { Home" }\end{array}$ \\
\hline Accessibility & $\mathrm{XXX}$ & $\mathrm{X}$ & -- \\
\hline Effectiveness & $\mathrm{X}$ & $\mathrm{XXX}$ & $\mathrm{XX}$ \\
\hline Attrition & $\mathrm{XXXX}$ & $\mathrm{X}$ & $\mathrm{XX}$ \\
\hline Integration & -- & $\mathrm{XXXX}$ & $\mathrm{XXX}$ \\
\hline Continuum & $\mathrm{X}$ & $\mathrm{XXXX}$ & $\mathrm{XXX}$ \\
\hline
\end{tabular}

it is associated with the irreducible issue of stigma, or comorbidities related with aging, as can be seen in current populations of people who have been infected with HIV for over twenty years.

b. Full adherence to antiretroviral treatment should be part of the HIV-depression remission criteria.

c. Depending on the mental health model to be implemented, there is the possibility of a better process outcome, so as not to interfere with the continuum of the effective and global treatment of HIV infection. In lowresource clinics in some countries, it can be very difficult to implement models of assistance based primarily on non-professional mental health workers ["Medical Home" type], due to cultural prejudices. The consultation-liaison model appears as the least appropriate, while the in-setting one, as the hardest to develop, because of the small number of trained psychiatrists within the HIV field.

Still, this last model is, in our opinion, the best option and we are therefore running it at this moment in our urban HIV clinic, with the aim of bridging the gap in the 90-90-90 program related to the HIV-depression burden. With the in-setting system, we see the integration of the three components of current HIV psychiatric assistance: psychiatric diagnosis and psychopharmacologic management (conducted by a psychiatrist); counseling and psychotherapy (conducted by a counselor or psychologist) and cognitive testing (conducted by a neuropsychologist). All of this, in close contact with the infectiology team.

We believe that this integrated model should be reproduced and tested in the HIV clinics in LMIC, in order to further advance the HIV-depression approach and ensure compliance with the 90-90-90 initiative.

\section{References}

1. Chibanda D. Depression and HIV: integrated care towards 90-90-90. Int Health 2017; 9: 77-79.

2. Corless IB, Hoyt AJ, Tyer-Viola L, et al. 90-90-90-Plus: Mantaining Adherence to Antiretroviral Therapies. AIDS Patient Care and STDs. 2017; 31: 227-235.

3. Pence BW, O'Donnell JK, Gaynes BN. Falling to the cracks: the gaps between depression prevalence, diagnosis, treatment, and response in HIV care (letter). AIDS. 2012; 26 (5): 636-637.

4. Cholera R, Pence Bw, Bengtson AM, et al. Mind the Gap in Antidepressant Treatment, Treatment Adjustments, and Outcomes among Patients in Routine HIV Care in a Multisite U.S. Clinical Cohort. PLOS ONE, 2017; 12(1): e0166435.

5. Gonzalez JS, Batchelder AW, Psaros C, et al. Depression and HIV/ AIDS Treatment Nonadherence: A Review and Meta-analysis. J Acquir Immune Defic Syndr. 2011; 58(2): 181-187.

6. Sin NL, DiMatteo MR. Depression Treatment Enhances Adherence to Antiretroviral Therpy: A Meta-Analysis. ann behav med. 2013. DOI 10.1007/s12160-013-9559-6.

7. Maccaferri GE, Cavassini M, Berney A. Mood disorders in HIV patients: a challenge for a liaison psychiatry consultation. Rev Med Suisse. 2012 Feb 15; 8(328): 362-4,366-7.

8. Dodds S, Nuhering EM, Blaney NT, et al. Integrating mental health services into primary HIV care for women: The Whole Life Project. Public Health Rep. 2004 Jan-Feb; 119(1): 48-59

9. Zirulnik JL, Perez HM. HIV psychiatry in the era of combined antirretroviral therapy: top five issues. J Int AIDS Soc. 2015; 18(1): 20725.

10. Adams JL, Gaynes BN, McGinness T, et al. Treating Depression Within the HIV "Medical Home". A Guided Algorithm for Antidepressant Management by HIV Clinicians. AIDS Patient Care and STDs. 2012; 26(11): 647-654. 